\title{
Case Report \\ Gastrointestinal Stromal Tumor in the Cecum of a Horse
}

\author{
S. Stephan, ${ }^{1}$ S. Hug, ${ }^{2}$ and M. Hilbe ${ }^{1}$ \\ ${ }^{1}$ Institute of Veterinary Pathology, Vetsuisse Faculty, University of Zurich, 8057 Zurich, Switzerland \\ ${ }^{2}$ Department of Equine Medicine, Vetsuisse Faculty, University of Zurich, 8057 Zurich, Switzerland \\ Correspondence should be addressed to S. Stephan, sara.stephan@laposte.net \\ Received 15 March 2012; Accepted 20 June 2012 \\ Academic Editors: J. Carmalt, C. Hyun, J. S. Munday, and F. Mutinelli
}

Copyright ( $) 2012$ S. Stephan et al. This is an open access article distributed under the Creative Commons Attribution License, which permits unrestricted use, distribution, and reproduction in any medium, provided the original work is properly cited.

Gastrointestinal stromal tumors (GISTs) are defined as specific CD117-(Kit, stem cell factor receptor) expressing tumors of the gastrointestinal (GI) tract. They are believed to originate from the interstitial pacemaker cells of Cajal (ICC) or their progenitor cells. In horses only a few cases of GISTs are described in the literature. In the present paper the macroscopical, histological, immunohistochemical, and ultrastructural features of an equine cecal GIST are described.

\section{Case History}

A 24-year-old, $540 \mathrm{~kg}$, warm-blood gelding was referred to the Internal Medicine Section of the Equine Department at the Vetsuisse Faculty of Zurich with colic. The horse was regularly vaccinated and dewormed. The horse had suffered from colic the previous week and had lost a lot of weight over the past few months. Five years before, the horse had undergone a colic surgery, where 5 meters of the small intestine had to be removed and an end-to-end anastomosis was performed. Since then it had been without clinical signs. On initial examination at the Vetsuisse Faculty of Zurich, the horse had a poor general condition. After rectal palpation, an impaction of the cranial colon ascendens was suspected and the horse was treated with dipyrone $(25 \mathrm{mg} / \mathrm{kg}$ iv $)$ and paraffinum liquidum as well as intravenous fluids. The next day the impaction was still present and the horse was treated further with hyperinfusion, moderate exercise, and was fasted. On the third day the gelding had recovered temporarily, but then relapsed and developed colic again. This time the medical treatment consisted of dipyrone ( $25 \mathrm{mg} / \mathrm{kg}$ iv), butylscopolamine (60 mg iv), and xylazine (120 mg iv). A hematological and biochemical blood examination, abdominocentesis, gastroscopy, and an ultrasonographic examination of the abdomen were initiated. The hematological blood examination showed a slight leukocytosis $(9,400 / \mu \mathrm{L} ; 4,700-8,200)$; the other parameters were within reference values. The biochemistry was unremarkable. The abdominal fluid was increased, yellow colored, and turbid with an increased cell count of $15,825 / \mu \mathrm{L}$, specific gravity 1019 , and protein $19 \mathrm{~g} / \mathrm{L}$. The cytological evaluation of the abdominal fluid showed a slight suppurative inflammation without neutrophilic degeneration. The gastroscopy showed no abnormal findings. On the ultrasonographic examination of the abdomen, two large heterogeneous solid masses of approximately 10 to $15 \mathrm{~cm}$ and $5 \mathrm{~cm}$ in diameter located in the mid and right caudal aspect of the abdomen most likely associated with the cecal wall could be seen. The suspected diagnosis of the cecal masses was a neoplasia (malignant lymphoma or carcinoma) and as a differential diagnosis an abscess formation or a granuloma. The horse was euthanized because of the poor prognosis and a necropsy was performed.

\section{Necropsy Findings}

Necropsy revealed two masses in the external cecal wall. One mass of approximately $10 \times 10 \times 8 \mathrm{~cm}$ was attached by a small stalk of about $1,5 \mathrm{~cm}$ in diameter approximately $10 \mathrm{~cm}$ distant to the apex region of the cecum located between the medial and ventral taenia of the cecum (Figure 1). The mass was oval shaped with a multilobulated surface and a red-beige-brown marmorated cross-section. The mass was soft, partially cystic, and of brittle consistency, separated by multifocal necrosis and hemorrhage (Figure 2). The second mass was of transmural $(5 \times 5 \times 8 \mathrm{~cm})$ and intraluminal $(4 \times$ $4 \times 3 \mathrm{~cm}$ ) growth, located in the corpus region of the cecum between the medial and lateral taenia. The nodule was of firm 


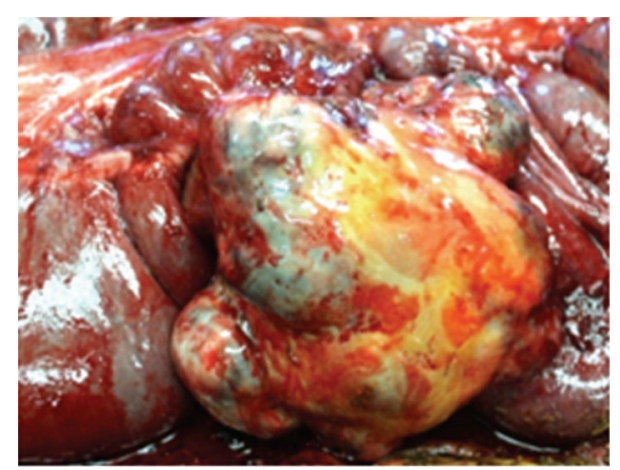

Figure 1: Necropsy revealed an approximately $10 \times 10 \times 8 \mathrm{~cm}$ neoplastic mass attached by a small stalk to the external cecal wall.

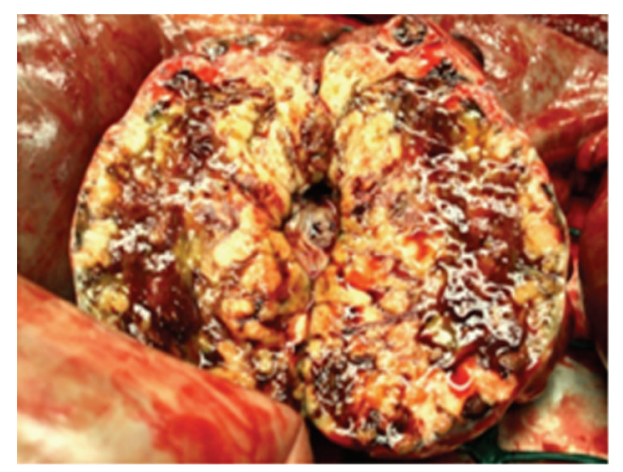

Figure 2: Cross-section: brittle consistency, red-beige mottled with multifocal necrosis/hemorrhage.

texture and of whitish color with central necrosis. Additional diagnostic finding consisted of an encapsulated foreign body (piece of wire) of $5 \mathrm{~cm}$ length located in the cranial part of the duodenum with secondary fistula formation. The other internal organs showed no macroscopic changes.

\section{Histologic, Immunohistochemical, and Electron Microscopic Findings}

Tissue samples of the cecum, the jejunum, the liver, and the tumor were fixed in $4 \%$ buffered-formalin processed conventionally and embedded in paraffin. Sections of about 2-3 $\mu \mathrm{m}$ were cut and stained with hematoxylin-eosin (HE) and periodic-acid-Schiff (PAS) and evaluated with light microscopy.

Histological examination revealed that the mass consisted of well-demarcated densely cellular tissue arranged in interlacing fascicles or in a storiform pattern with abundant fibrovascular tumor stroma and moderate mucinous matrix. The muscle layer of the cecal wall surrounded the extraluminal tumor part. Multifocal acute hemorrhages, edema, and necrosis were visible. Tumor cell populations showed slightto-moderate anisocytosis and anisokaryosis without mitosis. In the present case the tumor population was of mixed cell type with about 5\% epithelioid cells and about 95\% spindle cells. Cells of epithelioid morphology were roundto-polygonal-shaped with well-defined cell borders, roundto-oval nuclei, loosely arranged chromatin, and moderate eosinophilic cytoplasm. Spindle-shaped cells had indistinct cell borders, oval nuclei with dispersed granular heterochromatin, 1 to 2 small nucleoli, and scant fibrillar cytoplasm (Figures 3 and 4). The inflammatory response was slight and mainly composed of lymphocytes, few neutrophils, and some hemosiderin-loaded macrophages. Multifocal in between the cell groups and strands, some hyaline brightly bulked eosinophilic material could be found. These structures were PAS-positive aggregates of extracellular collagen bundles known as skeinoid fibers $[1,2]$. Additionally a severe focalextensive subacute to chronic lymphohistiocytic duodenitis with formation of moderate fibroangioblastic granulation tissue due to a foreign body could be diagnosed as an incidental finding.

Immunohistochemical staining was performed for CD117, vimentin, "alpha smooth muscle actin (SMA)," desmin, S100, "neuron specific enolase (NSE)," and Melan A. All sections, except for CD117 and desmin, were pretreated with a citrated ( $\mathrm{pH} \mathrm{6)}$ buffer for 20 minutes at $98^{\circ} \mathrm{C}$ by heating in a microwave oven. Section for desmin was pretreated in the same buffer only for 5 minutes at $98^{\circ} \mathrm{C}$, and section for CD117 was pretreated with an alkaline buffer $(\mathrm{pH} 9)$ for 20 minutes at $98^{\circ} \mathrm{C}$. For vimentin, SMA, desmin, S100, NSE, and melan A, the Detection-kit ChemMate from DAKO was used. For CD117 the OUM-kit from Roche was applied and performed on the discovery machine. Additionally, in all sections, endogenous peroxidase reaction was blocked with peroxidase-blocking solution (DAKO S2023). Antibodies from DAKO against the following antigens were used in the DAKO Autostainer (DAKO AG Switzerland): CD117 (rabbit, dilution $1: 400$, DAKO A4502), vimentin (mouse, dilution 1:100, DAKO M7020), SMA (prediluted mouse, DAKO N1584), desmin (prediluted mouse, DAKO N1526), S100 (prediluted rabbit, DAKO N1573), NSE (mouse, dilution 1:150, DAKO M087301), and Melan A (mouse, dilution 1:10, DAKO M719601). All antibodies were diluted with dilution buffer from DAKO (S2022). The sections were incubated with streptavidin-peroxidase conjugate and reacted with the substrate-chromogen 3-amino-9-ethylcarbazole (AEC, Detection-kit DAKO K 5003) at room temperature and counterstained with hematoxylin. Positive and negative controls were included. The neoplastic cells had moderate diffuse positive red intracytoplasmic granular staining for vimentin, showed strong diffuse pancytoplasmic homogenous to slight granular positive staining for CD117 (Figure 5), and were diffusely slight to moderate positive for NSE. In contrast, the tumor was negative for desmin, S100, and Melan A (Table 1).

For electron microscopy, one millimeter cubes of formalin-fixed and paraffin-embedded tumor locations were selected and further fixed in 2,5\% glutaraldehyde in $0,1 \mathrm{M}$ natriumphosphat buffer $(\mathrm{pH} 7,4)$. Postfixation was performed with $1 \%$ osmium tetroxide buffered in natriumphosphat. After dehydration in graded ethanol series, followed by propylenoxide, the samples were embedded in Epon resin. Ultrathin sections were stained with $2 \%$ uranyl acetate and 
TABLE 1: Slight staining $(+)$, moderate staining $(++)$, strong staining $(+++)$, no reaction $(-)$.

\begin{tabular}{lc}
\hline ICH & Results \\
\hline CD117 & +++ \\
Vimentin & +++ \\
SMA & - \\
Desmin & - \\
S100 & - \\
NSE & ++ \\
\hline
\end{tabular}

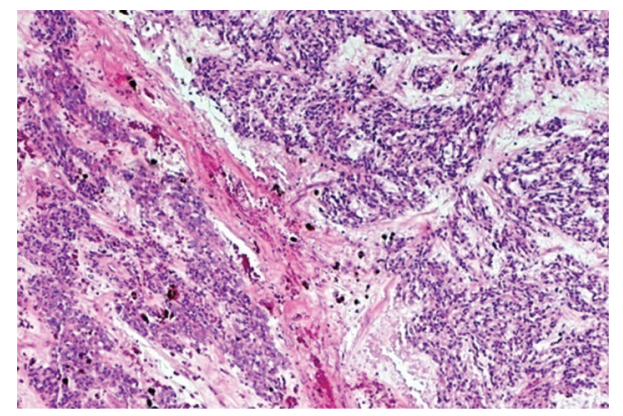

FIGURE 3: Predominant spindle-shaped tumor cells with a fascicular pattern. Spaces between tumor cell trabeculae are filled with erythrocytes, siderophages and collagenous (skeinoid fibers) as well as mucinous matrix (HE).

lead acetate. Samples were examined on a Philips CM 10 transmission electron microscope.

The tumor had a pleomorphic cell population with indistinct cell borders. The cells had round-to-elongated nuclei with marked nuclear lobulation and low-to-moderate peripheral packed chromatin. The cytoplasm contained variable swollen and dilatated mitochondria, low-to-moderate perinuclear smooth endoplasmic reticulum, and few neurosecretory granules $(150 \mathrm{~nm})$ which displayed an ultrastructural feature of neuronal differentiation. Foci of intermediate filaments and arrays of microtubules were detected. Furthermore, tumor cells were embedded in an amorphous matrix and were separated by moderate dense collagen fibers, interpreted as interstitial skeinoid fibers.

\section{Discussion}

GISTs are rather uncommon tumors in horses. To the author's knowledge, there are only five publications between 2001 and 2010 reporting on 15 GISTs in 14 horses. A former study in France could show that thoroughbred horses $(13,43 \%)$ and horses older than 10 years $(23,90 \%)$ were significantly more often affected by tumors in general and that the most frequent tumors were benign (adenomas, lipomas) [3]. Of the malignant tumors, the alimentary lymphoma is the most common intestinal neoplasia in horses, followed by adenocarcinoma and leiomyosarcoma most likely located in the small intestine, mainly in Arabian horses [4]. GISTs have been described for decades in humans and more recently

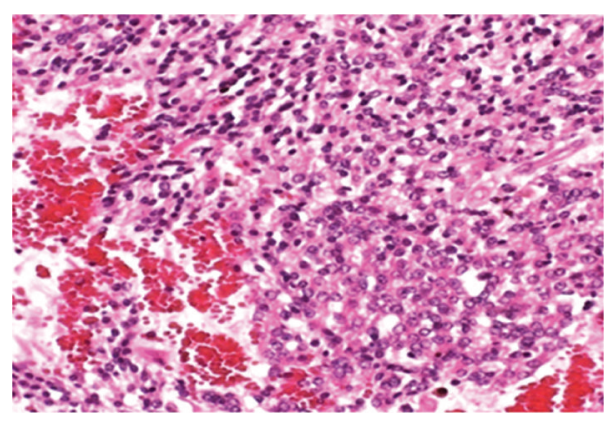

Figure 4: The distinction between epithelioid type (lower side right) and spindle cell type (upper side left) (HE).

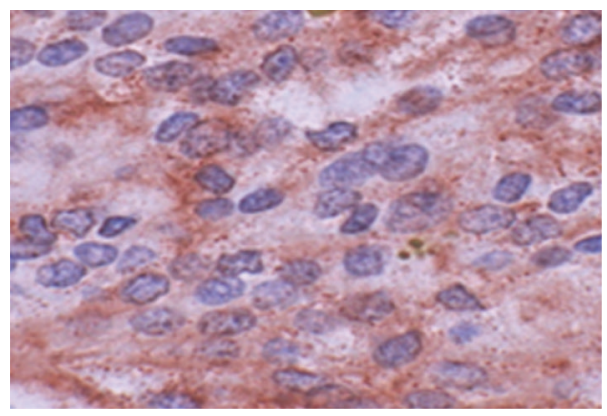

FIGURE 5: All tumor cells showed strong diffuse red pancytoplasmic homogenous to slight granular positive staining for CD117 (c-kit).

in dogs, nonhuman primates, horses, and rodents (rat and guinea pig) [5-9]. They are the most common primary nonlymphoid mesenchymal tumors of the GI tract with presumed histogenetic origin from the interstitial cells of Cajal (ICC) or of their pluripotent stem cells (precursorcell-hypothesis). Both are Kit-positive cells $[10,11]$. Kit is a transmembrane receptor tyrosine kinase encoded by the c-kit protooncogene and is nearly consistently expressed in all GISTs [12]. In addition, the embryonic isoform of smooth muscle myosin heavy chain is expressed in both GISTs and ICCs what supports the origin from ICCs [13]. Previously, these tumors were often misdiagnosed on morphologic basis as leiomyoma/-sarcomas, or schwannomas. Electron microscopic findings have demonstrated that most human GISTs derive from primitive mesenchymal cells that undergo either neurogenic, myogenic, dual, or no differentiation [14]. In the present case, without c-kit-staining, the tumor would have been diagnosed as a neuroendocrine tumor at histological level showing characteristics of neurogenic differentiation $(\mathrm{NSE}+)$. Ultrastructural studies revealed that a subgroup of GISTs seems to derive from the enteric plexi and is therefore called plexosarcoma or gastrointestinal autonomic nerve tumor (GANT) [15]. Most equine GISTs seem to be incidental findings at postmortem examination, but a few caused clinical signs like weight loss, chronic bleeding, anemia, and recurrent colic $[5,16]$. GIST development can be associated with gain-of-function mutations of c-kit, a protooncogene in humans that may lead to autophosphorylation, cellular proliferation, and reduction of apoptosis [17]. Actually, in horses 
no genetic investigations for GIST mutations are described in the literature. This would be important if therapies targeting the c-kit tyrosine kinase receptor, already available in human medicine, could be applied in horses. For tumor grading, the most consistent histologic features applied to predict malignancy are tumor size and mitotic index. Fletcher et al. in 2002 classified human GISTs into 4 risk categories: very low risk, low risk, intermediate risk, and high risk based on tumor size and mitotic counting [21]. Applying the human classification, the present tumor would be categorized into a high-risk tumor because of a size of $>10 \mathrm{~cm}$ and no mitotic figures. Other criteria of malignancy were present in this tumor-like marked cellular pleomorphism, pronounced anisokaryosis, invasiveness, hemorrhage, and necrosis, but it still remains difficult to provide an accurate prognosis for animals [8]. The location of the tumor may also be an important prognostic factor in human GISTs where a significant difference in site-specific survival could be shown. The survival curve was very good for GISTs located in the stomach. In contrast, small-bowel tumors showed a much poorer prognosis [18]. In horses it depends primarily on the degree of expansive tumor growth that may result in intestinal obstruction and colic. In the present case, the tumor was located in the apex and corpus region of the cecum. In a recent study from Hafner et al. in 1998, most of nine GISTs from seven horses investigated were also located in the distal region of the cecum. Thus, the cecum appears to be a common location in horses and dogs $[5,8,16]$. Curiously, human GISTs occur more often in the stomach followed by the small intestine [19], and it is interesting to note that five of seven GISTs in nonhuman primates have also been found in the stomach [6]. In addition the present tumor was of multiple incidence, which is in contrast to a recent study where almost all equine GISTs were solitary and without detected metastasis in necropsy [16]. However multiple tumors have been described in old horses, dogs, and cats, but metastases were rare [14]. Thus it is difficult to decide whether multifocal tumors may be interpreted as metastasis or multiple tumor formation. Metastatic GISTs are described in the literature. In a previous study metastatic nodules were found in the liver, the spleen, the femur, and the bone marrow of a F344 rat [7] and widespread abdominal metastasis were diagnosed in a rhesus macaque [20]. Typical of malignant human GISTs is intra-abdominal spread and distant metastases most commonly to the liver followed by the lung and the bone [18]. Furthermore, vimentin expression is correlated with a light microscopic diagnosis of malignancy. Thus immunohistochemical staining for vimentin may be useful in more accurately defining tumors of questionable malignant behavior [8]. GISTs that are considered as tumors of malignant behavior need to be surgically removed. Segmental resection or complete en bloc resection is the standard treatment in human and equine medicine. Inoperable or metastatic human GISTs are treated with imatinib mesylate a competitive inhibitor of c-kit (CD117) [21]. Tumors without CD117 expression but with similar morphologic features are called GIST-like tumors [22]. There was no immunohistochemistry performed on CD34 (hematopoietic progenitor cell antigen), commonly immunopositive in human GISTs and in ICCs, because the antibody did not stain equine control tissue in a recent study [16]. Besides, in a nonhuman primate none of the neoplastic cells were immunoreactive for CD34 [7]. The SMA positivity of human GISTs is about $30-40 \%$ and is correlated with a more favorable prognosis in gastric and small intestine tumors. In contrast, desmin is rare in human GISTs of all sites [23]. In the present case, the staining was negative for both implying no myogenic differentiation of the tumor cells. This is in accordance to previous studies on equine GISTs $[5,16]$. As human GISTs, equine tumors occur in mature adults and more often in mares [5], but case numbers are limited. Finally in the present case, the foreign body located in the cranial part of the duodenum might also have caused digestion disturbance and recurrent colic, so that we cannot explain whether the foreign body, the GIST, or both had caused the clinical signs. In summary, the tumor presented here was diagnosed as a GIST because of positive expression of CD117 and vimentin, immunonegativity for desmin, and variable staining features for SMA, S100, and NSE depending on the tumor differentiation. Immunohistochemical and ultrastructural features suggested a tumor of the neural type according to the classification from Rosai in 1996 [24]. This study demonstrated once more the necessity of immunohistochemical staining of c-kit to support the diagnosis of a GIST.

\section{Conflict of Interests}

The authors declared that they had no conflict of interests with respect to their authorship or the publication of this paper.

\section{Funding}

The authors declared that they received no financial support for their research and/or authorship of this paper.

\section{Acknowledgments}

The authors thank the whole laboratory staff of the Institute of Veterinary Pathology of the Vetsuisse Faculty of Zurich for excellent technical work. They are also grateful to the Department of Equine Medicine of the Vetsuisse Faculty of Zurich for providing clinical and laboratory findings.

\section{References}

[1] K. W. Min, "Small intestinal stromal tumors with skeinoid fibers: clinicopathological, immunohistochemical, and ultrastructural investigations," American Journal of Surgical Pathology, vol. 16, no. 2, pp. 145-155, 1992.

[2] S. Matsukuma, M. Doi, M. Suzuki, K. Ikegawa, K. Sato, and N. Kuwabara, "Numerous eosinophilic globules (skeinoid fibers) in a duodenal stromal tumor: an exceptional case showing smooth muscle differentiation," Pathology International, vol. 47, no. 11, pp. 789-793, 1997. 
[3] C. Laugier, N. Doux, C. George et al., "Prévalence de la pathologie tumorale dans un effectif de 1771 chevaux autopsiés," Pratique Vétérinaire Equine, vol. 143, pp. 21-35, 2004.

[4] S. D. Taylor, N. Pusterla, B. Vaughan, M. B. Whitcomb, and W. D. Wilson, "Intestinal neoplasia in horses," Journal of Veterinary Internal Medicine, vol. 20, no. 6, pp. 1429-1436, 2006.

[5] F. Del Piero, B. A. Summers, J. F. Cummings, G. Mandelli, and E. A. Blomme, "Gastrointestinal stromal tumors in equids," Veterinary Pathology, vol. 38, no. 6, pp. 689-697, 2001.

[6] Y. R. Bommineni, E. J. Dick, and G. B. Hubbard, "Gastrointestinal stromal tumors in a baboon, a spider monkey, and a chimpanzee and a review of the literature," Journal of Medical Primatology, vol. 38, no. 3, pp. 199-203, 2009.

[7] H. Fujimoto, M. Shibutani, K. Kuroiwa et al., "A case report of a spontaneous gastrointestinal stromal tumor (GIST) occurring in a F344 rat," Toxicologic Pathology, vol. 34, no. 2, pp. 164-167, 2006.

[8] R. G. Larock and P. E. Ginn, "Immunohistochemical staining characteristics of canine gastrointestinal stromal tumors," Veterinary Pathology, vol. 34, no. 4, pp. 303-311, 1997.

[9] H. Bielefeldt-Ohmann, D. H. Barouch, A. M. Bakke, A. G. Bruce, M. Durning, and R. Grant, "Intestinal stromal tumors in a simian immunodeficiency virus-infected, simian retrovirus-2 negative rhesus macaque (Macaca mulatta)," Veterinary Pathology, vol. 42, no. 3, pp. 391-396, 2005.

[10] L. G. Kindblom, H. E. Remotti, F. Aldenborg, and J. M. MeisKindblom, "Gastrointestinal pacemaker cell tumor (GIPACT): gastrointestinal stromal tumors show phenotypic characteristics of the interstitial cells of Cajal," The American Journal of Pathology, vol. 152, no. 5, pp. 1259-1269, 1998.

[11] M. Miettinen, J. M. Monihan, M. Sarlomo-Rikala et al., "Gastrointestinal stromal tumors/smooth muscle tumors (GISTs) primary in the omentum and mesentery: clinicopathologic and immunohistochemical study of 26 cases," The American Journal of Surgical Pathology, vol. 23, no. 9, pp. 1109-1118, 1999.

[12] H. Joensuu, C. Fletcher, S. Dimitrijevic, S. Silberman, P. Roberts, and G. Demetri, "Management of malignant gastrointestinal stromal tumours," The Lancet Oncology, vol. 3, no. 11, pp. 655-664, 2002.

[13] S. Sakurai, T. Fukasawa, J. M. Chong, A. Tanaka, and M. Fukayama, "Embryonic form of smooth muscle myosin heavy chain (SMemb/MHC-B) in gastrointestinal stromal tumor and interstitial cells of Cajal," The American Journal of Pathology, vol. 154, no. 1, pp. 23-28, 1999.

[14] K. W. Head, J. M. Cullen, P. R. Dubielzig et al., Histological Classification of Tumors of the Alimentary System of Domestic Animals, vol. 10 of International Histological Classification of Tumors of Domestic Animals, WHO, Armed Forces Institute of Pathology, Washington, DC, USA, 2003.

[15] G. Isimbaldi, M. Santangelo, G. Cenacchi et al., "Gastrointestinal autonomic nerve tumor (Plexosarcoma): report of a case with fine needle aspiration biopsy and histologic, immunocytochemical and ultrastructural study," Acta Cytologica, vol. 42, no. 5, pp. 1189-1194, 1998.

[16] S. Hafner, B. G. Harmon, and T. King, "Gastrointestinal stromal tumors of the equine cecum," Veterinary Pathology, vol. 38, no. 2, pp. 242-246, 2001.

[17] S. Hirota, K. Isozaki, Y. Moriyama et al., "Gain-of-function mutations of C-kit in human gastrointestinal stromal tumors," Science, vol. 279, no. 5350, pp. 577-580, 1998.
[18] T. S. Emory, L. H. Sobin, L. Lukes, D. H. Lee, and T. J. O'Leary, "Prognosis of gastrointestinal smooth-muscle (stromal) tumors: dependence on anatomic site," The American Journal of Surgical Pathology, vol. 23, no. 1, pp. 82-87, 1999.

[19] M. Miettinen and J. Lasota, "Gastrointestinal stromal tumors-definition, clinical, histological, immunohistochemical, and molecular genetic features and differential diagnosis," Virchows Archiv, vol. 438, no. 1, pp. 1-12, 2001.

[20] M. Banerjee, L. J. Lowenstine, and R. J. Munn, "Gastric stromal tumors in two rhesus macaques (Macaca mulatta)," Veterinary Pathology, vol. 28, no. 1, pp. 30-36, 1991.

[21] C. D. M. Fletcher, J. J. Berman, C. Corless et al., "Diagnosis of gastrointestinal stromal tumors: a consensus approach," Human Pathology, vol. 33, no. 5, pp. 459-465, 2002.

[22] M. V. C. De Silva and R. Reid, "Gastrointestinal stromal tumors (GIST): C-kit mutations, CD117 expression, differential diagnosis and targeted cancer therapy with imatinib," Pathology and Oncology Research, vol. 9, no. 1, pp. 13-19, 2003.

[23] M. Miettinen and J. Lasota, "Gastrointestinal stromal tumors: review on morphology, molecular pathology, prognosis, and differential diagnosis," Archives of Pathology and Laboratory Medicine, vol. 130, no. 10, pp. 1466-1478, 2006.

[24] J. Rosai, "Stromal tumors," in Ackerman's Surgical Pathology, J. Rosai, Ed., vol. 8, pp. 691-693, Mosby, St. Louis, Mo, USA, 1996. 

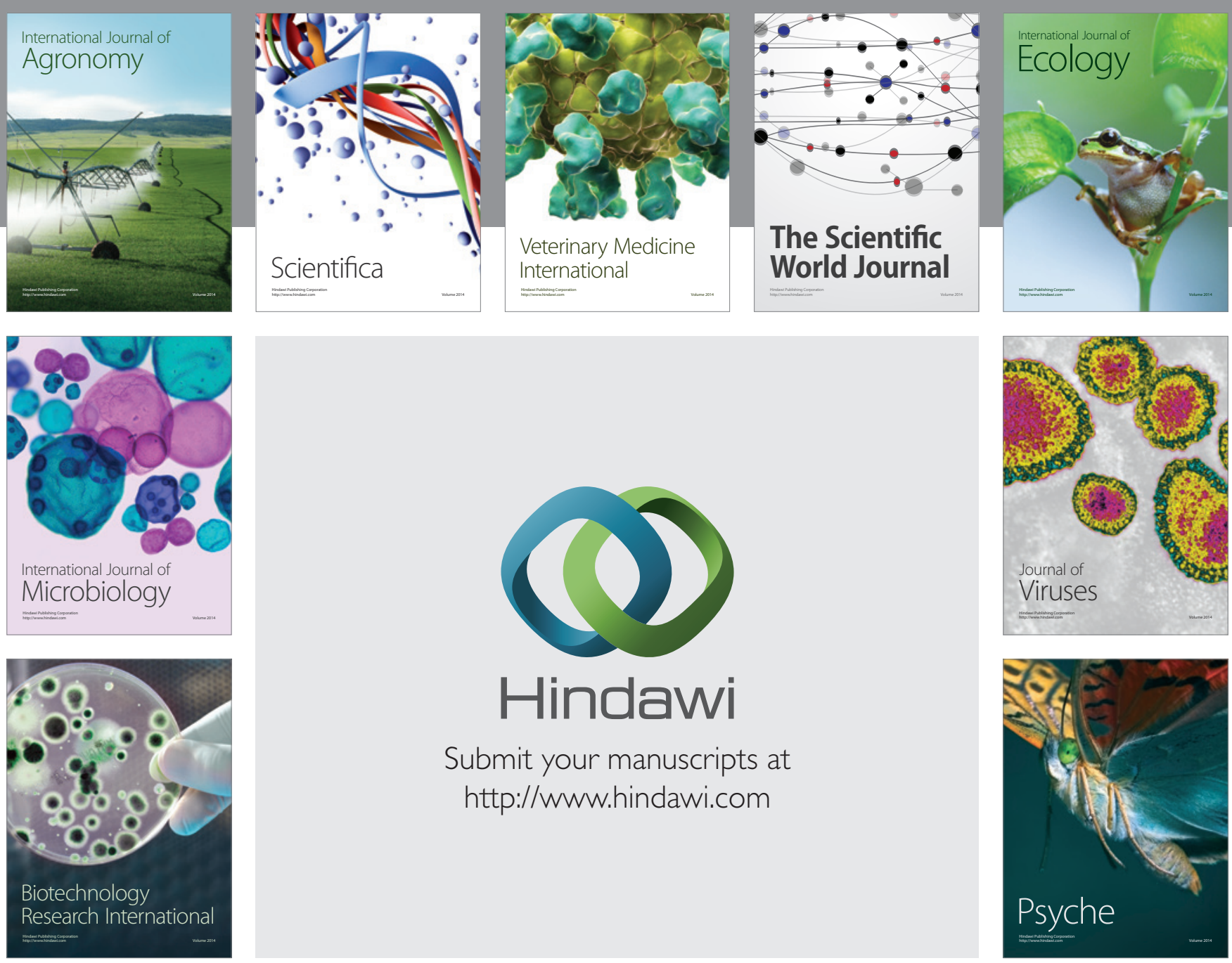

Submit your manuscripts at

http://www.hindawi.com
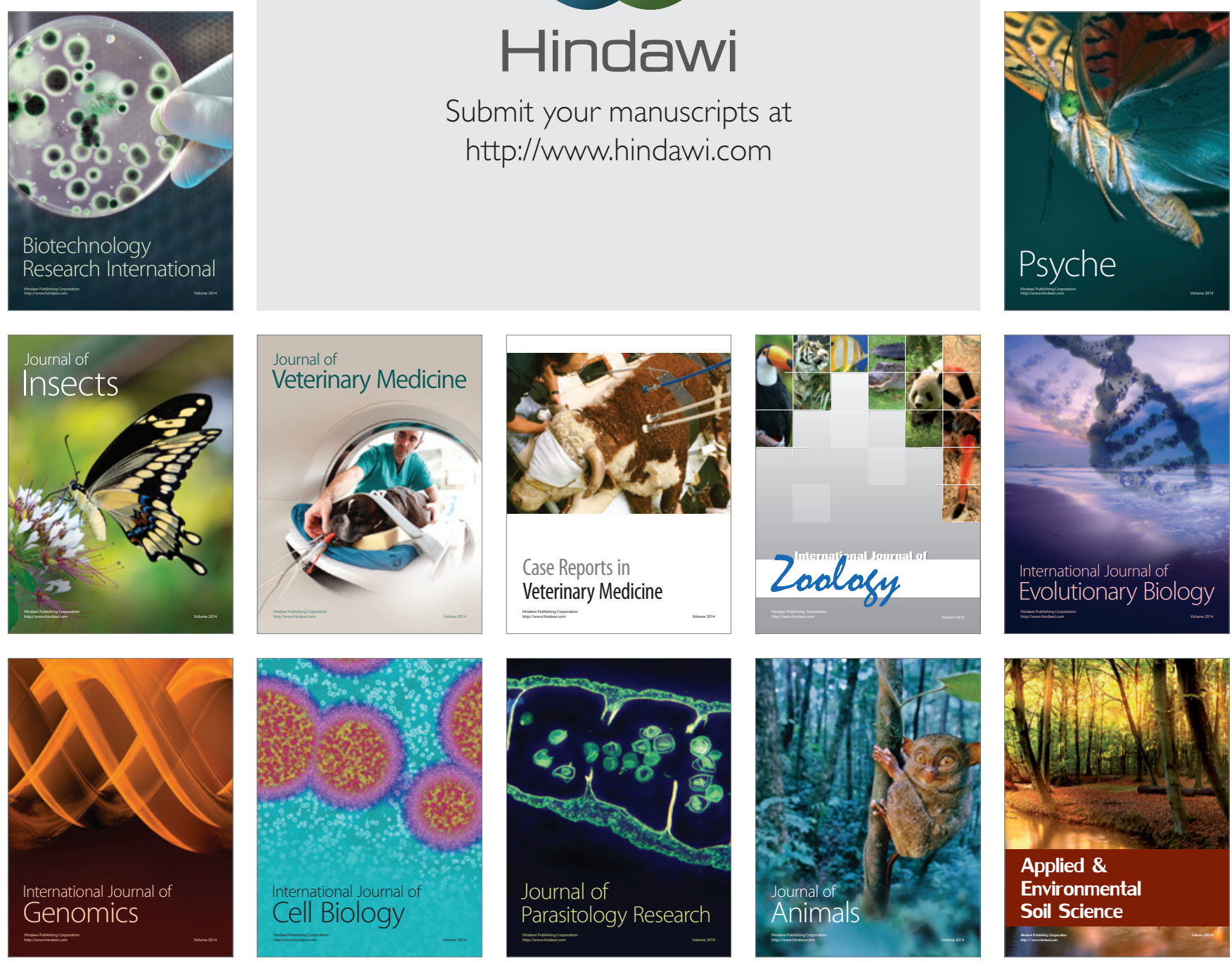